\title{
A clinical and molecular analysis of a patient with Emanuel syndrome
}

\author{
JIN-WEN LUO ${ }^{1}$, HUAN YANG $^{2}$, ZHI-PING TAN ${ }^{3}$, MING TU $^{4}$, HONG LUO $^{5}$, YI-FENG YANG ${ }^{6}$ and LI XIE $^{6}$ \\ ${ }^{1}$ Department of Cardio-Thoracic Surgery, Hunan Children's Hospital, Changsha, Hunan 410007; \\ ${ }^{2}$ Department of Respiratory Medicine, Hunan Provincial People's Hospital, Changsha, Hunan 410001; \\ ${ }^{3}$ The Clinical Center for Gene Diagnosis and Therapy of The State Key Laboratory of Medical Genetics, \\ The Second Xiangya Hospital of Central South University, Changsha, Hunan 410011; \\ ${ }^{4}$ The Laboratory of Genetics and Metabolism, Hunan Children's Hospital, Changsha, Hunan 410007; \\ Departments of ${ }^{5}$ Respiratory Medicine and ${ }^{6}$ Cardiovascular Surgery, \\ The Second Xiangya Hospital of Central South University, Changsha, Hunan 410011, P.R. China
}

Received November 21, 2015; Accepted December 1, 2016

DOI: $10.3892 / \mathrm{mmr} .2017 .6107$

\begin{abstract}
Emanuel syndrome (ES) is the most frequent type of recurrent non-Robertsonian translocation that is characterized by numerous anomalies. Over 100 patients with ES have been described in the literature. The phenotype of this syndrome varies but often consists of facial dysmorphism, microcephaly, severe intellectual disability, developmental retardation, congenital heart disease and genital anomalies. The present study describes a 2-year-old boy with multiple malformations, including facial dysmorphism, severe intellectual disability, growth retardation, congenital heart disease, cleft lip and palate, genital malformation (micropenis), amblyopia, thymic dysplasia and hearing impairment. The karyotype of the patient was $47, X Y,+\operatorname{del}(22)(q 13)$, and the maternal karyotype was 46,XX,t(11;22)(q25;q13),9qh-,15p+. Single-nucleotide polymorphism-array analysis of the proband indicated a partial duplication of chromosomes 22 and 11 at 22q11.1-q11.21 and 11q23.3-q25, respectively, which confirmed the diagnosis of ES. To date, no cases of ES have been reported in mainland China. The present case further emphasizes the necessity and importance of high-resolution techniques for genetic diagnosis and for subsequent genetic counseling. The present study contributed to the phenotypic delineation of ES and confirmed the first ES patient in mainland China.
\end{abstract}

Correspondence to: $\mathrm{Dr} \mathrm{Li}$ Xie, Department of Cardiovascular Surgery, The Second Xiangya Hospital of Central South University, 139 Renmin Road, Changsha, Hunan 410011, P.R. China

E-mail: csuxieli@163.com

Key words: Emanuel syndrome, single-nucleotide polymorphism array, copy number variants, translocation, congenital heart disease

\section{Introduction}

Emanuel syndrome (ES), also referred to as derivative 22 syndrome, derivative 11;22 syndrome, partial trisomy $11 ; 22$, or supernumerary $\operatorname{der}(22) \mathrm{t}(11 ; 22)$ syndrome $(1,2)$, is an unbalanced translocation syndrome that usually results from a 3:1 meiotic disjunction of a parental balanced translocation between chromosomes 11 and 22 during gametogenesis. In $>99 \%$ of patients with ES, one of the parents is a balanced carrier of a $\mathrm{t}(11 ; 22)(\mathrm{q} 23 ; \mathrm{q} 11.2)$ translocation and normal phenotype. The possible outcomes of future pregnancies of the parents include: Normal chromosomes, Emanuel syndrome, balanced $\mathrm{t}(11 ; 22)$ carrier, and spontaneous abortion as a result of ES or another meiotic malsegregation. ES is characterized by severe intellectual disability, microcephaly, failure to thrive, preauricular tag or sinus, ear anomalies, cleft or high-arched palate, micrognathia, congenital heart disease, anal atresia, diaphragmatic hernia and genital abnormalities in men (3). The prevalence of ES is estimated at 1 in 110,000, and long-term survival is possible (4,5). Referring to the treatment strategies of ES, care provided by a multidisciplinary team is essential, and includes standard management of anal atresia, diaphragmatic hernia, cardiac defects, hearing loss, cleft palate and speech therapies.

Clinical diagnosis of ES depends on the distinct phenotype above. The molecular genetic testing foe ES consists of chromosome analysis, fluorescence in situ hybridization, chromosomal microarray analysis. In the present study, chromosome analysis and chromosomal microarray analysis, which usually include comparative genetic hybridization and single nucleotide polymorphism (SNP)-arrays, were performed. Chromosomal microarray analysis has greatly improved the available chromosome resolution and the identification of chromosomal abnormalities, particularly in defining the breakpoint of rearrangements and the accurate detection of copy number variants. The present study aimed to confirm the first patient with ES in China, and to promote the understanding of this syndrome in mainland China. 


\section{Materials and methods}

Ethical approval and patient consent. This study was approved by the Review Board of The Second Xiangya Hospital of Central South University (Changsha, China). Written informed consent was obtained from the parents of the patient for publication of this study and any accompanying images.

Clinical presentation. The proband, a 2-year-old boy, was born full term to a 26-year-old G1P1 mother and a 30-year-old father. The family history is unremarkable. The mother had a history of threatened abortion at 8 weeks gestation and underwent tocolytic treatment at a local hospital. At 5 months old, the patient received surgery to repair a cleft lip; at this time, a cardiac murmur was identified and echocardiography revealed an atrial septal defect (ASD). The child was referred to The Second Xiangya Hospital of Central South University (Changsha, China) and the ASD was repaired in December 2014; however, during surgery, thymic dysplasia was revealed. Karyotype analysis determined the proband to be $47, \mathrm{XY},+$ del (22)(q13) and the maternal karyotype was $46, \mathrm{XX}, \mathrm{t}(11 ; 22)$ (q25;q13),9qh-,15p+. During hospitalization, it was noted that the patient was unable to speak and walk. The Gesell Maturation Scale determined a developmental quotient of 33, which indicates an intelligence level equal to a 30-week-old infant, and is attributed to the severe mental and developmental retardation of the patient. Hearing impairment was detected using an auditory acuity test. Physical examination revealed facial dysmorphism, cleft lip and palate, genital malformation (micropenis) and amblyopia. Dysmorphic features included a prominent forehead, widely separated eyes with down-slanting palpebral fissure, broad nasal bridge, prominent philtrum, and bilateral large and low-set ears (Fig. 1).

Follow-up was completed in September 2015; at this time echocardiography revealed no left-to-right shunt at the atrial septum, and the ejection fraction was normal.

Cytogenetic analysis. Peripheral blood $(5 \mathrm{ml})$ was collected from the patient and each of the parents, and chromosome analysis was performed by conventional G-banding techniques (550-band resolution). All samples were subjected to lymphocyte culture according to standard cytogenetic protocol (6).

SNP-array analysis. Genomic DNA was isolated from peripheral blood of the patient and the two parents using the DNeasy Blood \& Tissue kit (Qiagen, Inc., Valencia, CA, USA) with the QIAcube automated DNA extraction robot (Qiagen $\mathrm{GmbH}$, Hilden, Germany). Genomic DNA samples were adjusted to a final concentration of $50 \mathrm{ng} / \mathrm{ml}$. The HumanOmni1-Quad BeadChip (Illumina, Inc., San Diego, CA, USA) and the Illumina BeadScan genotyping system (Beadstation Scanner; Illumina, Inc.) were employed to obtain the signal intensities of the SNP probes (7-9). The HumanOmnil-Quad Beadchip contains $>1.1$ million loci across the human genome, including markers derived from the International HapMap Project, the 1000 Genomes Project (http://www.internationalgenome.org/) and previously published studies (10). The human genome assembly GRCh37/hg19 from the University of California, Santa Cruz Genome Browser (Santa Cruz, CA, USA) was used with Illumina GenomeStudio v2011 (Illumina, Inc.) software to analyze the genotypes and to evaluate the experimental quality. The call rates of the samples are $>99.2 \%$.

\section{Results}

Chromosome analysis of the proband and his parents was performed using G-banding techniques on stimulated blood lymphocytes. Cytogenetics revealed the karyotypes of the proband and his mother to be 47,XY,+del(22)(q13) and 46,XX,t(11;22)(q25;q13),9qh-,15p+, respectively (Fig. 2); his father had a normal karyotype result.

To define the chromosomal abnormality, SNP-array analysis was performed on the proband's genomic DNA using the Illumina HumanOmni1-Quad array (Fig. 3). This analysis revealed a region of gain that spanned $\sim 3.1 \mathrm{Mb}$ on chromosome 22q11.1-q11.21, between 16,855,618 and 19,995,480 bp (Fig. 3B). In addition to the aforementioned duplication, the present study detected a duplication of $\sim 18.2 \mathrm{Mb}$ in chromosome region 11q23.3-q25, between 116,696,681 and 134,942,926 bp (Fig. 3A). The diagnosis of ES was confirmed by comparing the breakpoints identified in this patient with those reported in other cases of ES (11). Maternal SNP-array analysis was normal.

\section{Discussion}

ES is an inherited chromosomal abnormality syndrome (3) that consists of a derivative chromosome 22 as a supernumerary chromosome with a karyotype of $47, \mathrm{XX},+\operatorname{der}(22) \mathrm{t}(11 ; 22)$ (q23;q11) in females or $47, X Y,+\operatorname{der}(22) t(11 ; 22)(q 23 ; q 11)$ in males (5). In 2004, this chromosomal imbalance was named as ES (OMIM no. 609029) (1,3) According to previous reports, $>100$ individuals with ES have been reported (1,3,12-16); however, the patient in the present study is the first, to the best of our knowledge, to be reported in China.

Patients with ES exhibit a distinctive phenotype, and global developmental delay is observed in almost all patients. Congenital heart disease is present in $\sim 57 \%$ subjects, with the three most common anomalies being ASD, ventricular septal defect and patent ductus arteriosus. Other heart malformations, including tetralogy of Fallot, coarctation of the aorta, pulmonic stenosis, total anomalous pulmonary venous connection, truncus arteriosus, transposition of the great arteries and tricuspid atresia, have previously been reported (1,5,16-18). In the present study, besides ASD, the proband presented with severe intellectual disability and developmental retardation, congenital heart disease, facial dysmorphism, cleft lip and palate, thymic dysplasia, hearing impairment, genital malformation (micropenis) and amblyopia; these clinical features have been observed in other patients with ES.

Based on the clinical features of the proband and the karyotype of his mother, the diagnosis of ES was considered, but not confirmed, following karyotype analysis. Translocation-specific polymerase chain reaction (PCR) is the most cost-effective diagnostic method for $\operatorname{der}(22) \mathrm{t}(11 ; 22)$ and $\operatorname{der}(22) \mathrm{t}(8 ; 22)$ (19); however, other chromosomal abnormalities cannot be detected using this method. For this reason, the more accurate and precise SNP-array is used to verify the results of translocation-specific PCR. Therefore, SNP-array analysis was considered to further investigate the genetic variation of 
A

B

C

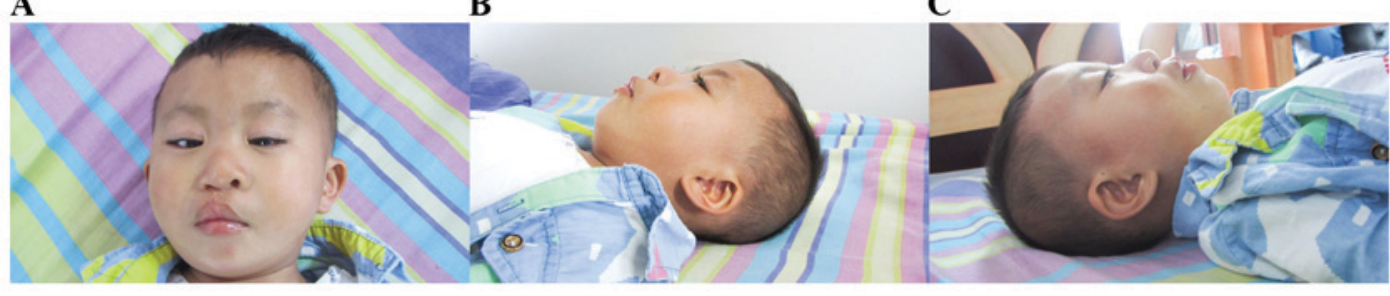

Figure 1. Facial features of the proband. (A) Frontal and (B and C) lateral views of the patient. (A) The patient has a prominent forehead, widely separated eyes with downslanting palpebral fissure, broad nasal bridge and prominent philtrum. (B and C) The patient has large and low-set ears.

A

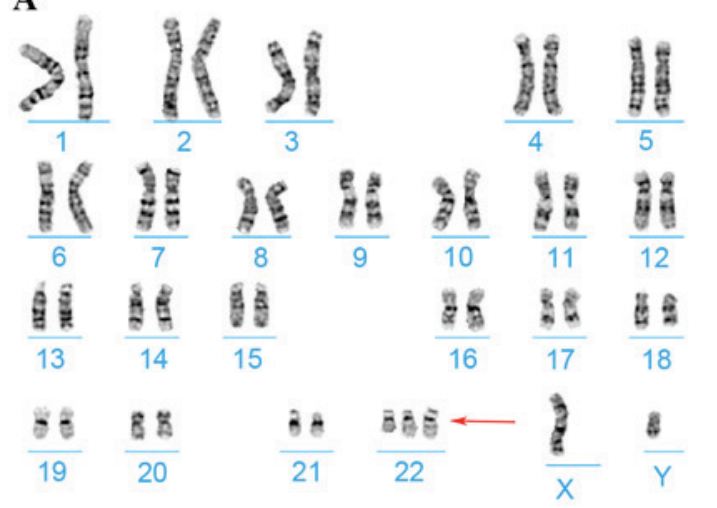

B

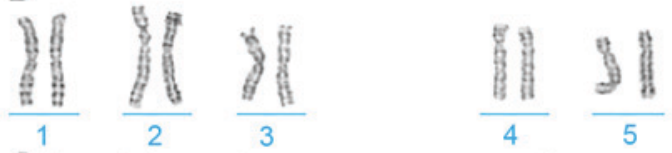

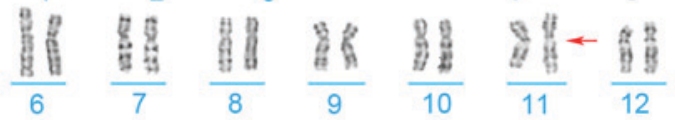

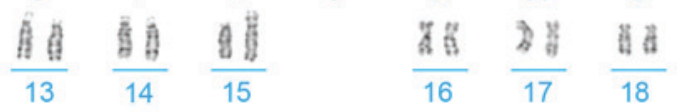

$\frac{8:}{19} \quad \frac{8:}{20}$

$\frac{5 \text { B }}{21} \quad \frac{58}{22} \leftarrow$

$\frac{18}{X} \frac{}{Y}$

Figure 2. Results of karyotype analysis. (A) G-banded chromosomes revealing 47,XY,+del(22)(q13) in the patient; red arrow indicates partial trisomy of chromosome 22. (B) G-banded chromosomes revealing 46,XX,t(11;22)(q25;q13),9qh-,15p+ in the patient's mother; red arrow indicates partial translocation of chromosome 11 and 22 .

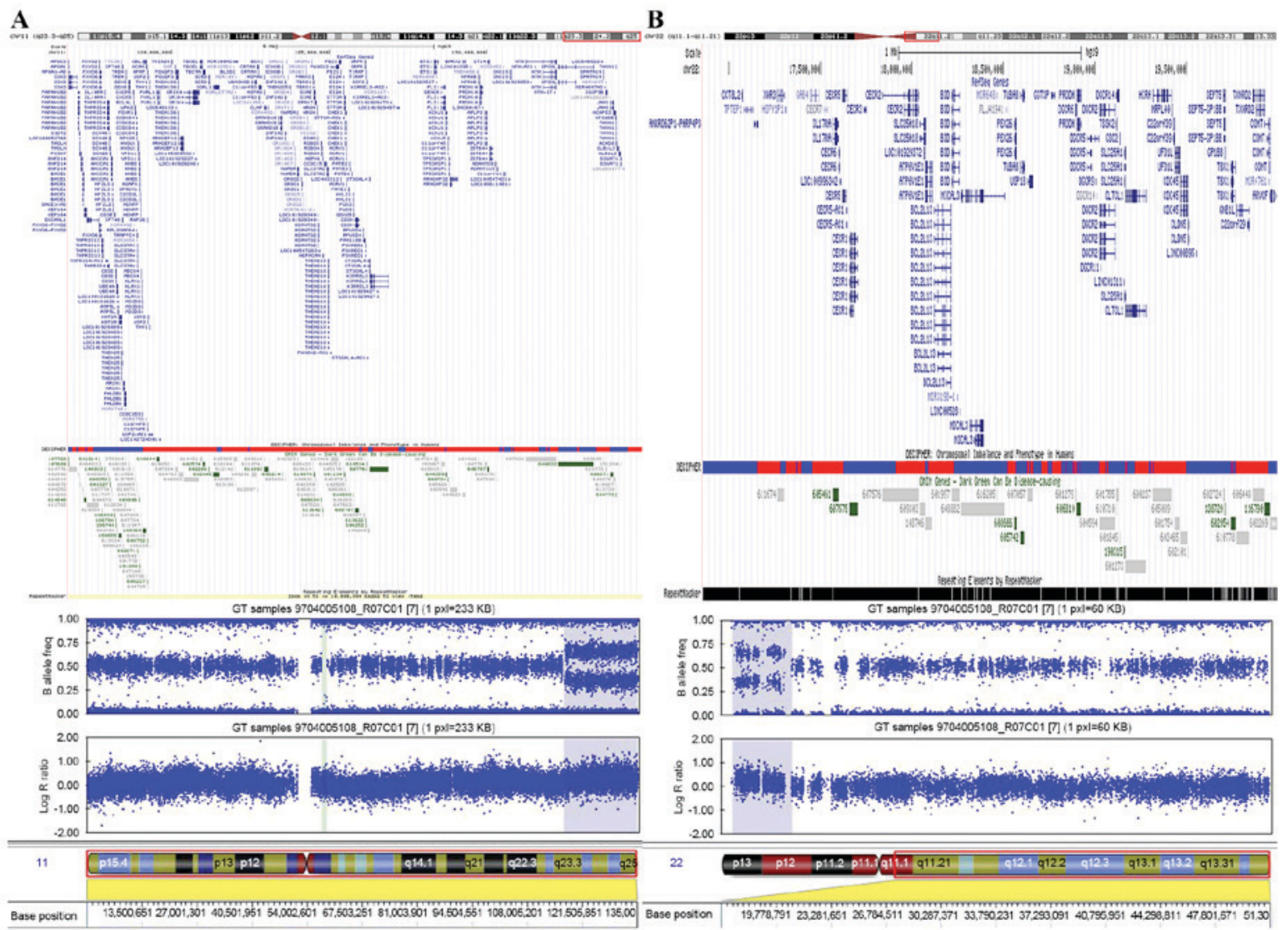

Figure 3. Illumina SNP-array results of the proband. (A) HumanOmni1-Quad SNP-array analysis of the 11q23.3-q25 region indicates a $18.2 \mathrm{Mb}$ duplication (Chr11: 116,696,681-134,942,926). (B) HumanOmni1-Quad SNP-array analysis of the 22q11.1-q11.21 region indicates a $3.1 \mathrm{Mb}$ duplication (Chr22: 16,855,618-19,995,480 bp). SNP, single-nucleotide polymorphism. 
the proband. SNP-array analysis identified two pathogenic duplications, which involved 22q11.1-q11.21 (3.1Mb) and 11q23.3-q25 (18.2Mb), respectively. This result confirmed the diagnosis of ES and demonstrated the limit of karyotype analysis, suggesting that a high-resolution technique, such as the SNP-array or array comparative genomic hybridization, should be recommended when karyotype analysis is uncertain.

Duplication of $22 \mathrm{q} 11.2$ has been reported to present a highly variable phenotype that ranges from healthy individuals to those with learning disabilities and congenital defects (20-23). Some of the main clinical features of $22 q 11.2$ duplication, including facial dysmorphism, hearing impairment and congenital heart disease, were present in the patient in the present study. One crucial gene that is affected by $22 \mathrm{q} 11.2$ duplication is T-box 1, which contributes to the phenotype of the proband with ES.

Duplication of 11q23.3-q25 without a copy number change of another chromosome is rare (24-28) and shares several common clinical features with 22q11.2 duplication, such as intellectual disability, growth retardation, microcephaly, facial dysmorphism, epilepsy, congenital inguinal hernia, and cardiac, renal and cerebral malformations (27,29), some of which were presented in the patient in the present study. The duplication region of this patient contains >100 OMIM genes, many of which have not been well characterized. The genes that contribute to the phenotypes of 11q23.3-q25 duplication and the mechanisms by which changes in gene dosage exert disruptive effects on gene structure and function remain unknown. Above all, the clinical phenotype of ES arises from the duplication of 22q11 and 11q23-qter (25).

When the mother is a balanced translocation carrier, there is an increased sibling recurrence-risk ratio of children with ES, as well as pregnancy loss due to other types of unbalanced translocations. Genetic counseling is essential and indispensable for this population. Furthermore, prenatal diagnosis could be performed through chorionic villus biopsy or amniocentesis.

In conclusion, the present study described the clinical features of a patient with ES. Although $>100$ patients with ES have been reported worldwide, the proband in the present study is the first, to the best of our knowledge, to be reported in China. Considering the prevalence of ES and the population of China, there are likely to be numerous patients with undiagnosed ES. The present study emphasizes the necessity and importance of high-resolution techniques for the molecular diagnosis of chromosomal abnormalities, and contributes to the further mapping and phenotypic delineation of ES.

\section{Acknowledgements}

The authors of the present study would like to thank the State Key Laboratory of Medical Genetics of China for technical assistance. This study was supported by the National Natural Science Foundation of China (grant no. 81500245 to LX, grant no. 81101475 to ZPT, and grant no. 81370204 to YFY).

\section{References}

1. Fraccaro M, Lindsten J, Ford CE and Iselius L: The 11q;22q translocation: A European collaborative analysis of 43 cases. Hum Genet 56: 21-51, 1980.
2. Hou JW: Supernumerary chromosome marker Der(22)t(11;22) resulting from a maternal balanced translocation. Chang Gung Med J 26: 48-52, 2003.

3. Zackai EH and Emanuel BS: Site-specific reciprocal translocation, $\mathrm{t}(11 ; 22)(\mathrm{q} 23 ; \mathrm{q} 11)$, in several unrelated families with $3: 1$ meiotic disjunction. Am J Med Genet 7: 507-521, 1980.

4. Ohye T, Inagaki H, Kato T, Tsutsumi M and Kurahashi H: Prevalence of Emanuel syndrome: Theoretical frequency and surveillance result. Pediatr Int 56: 462-466, 2014.

5. Carter MT, St Pierre SA, Zackai EH, Emanuel BS and Boycott KM: Phenotypic delineation of Emanuel syndrome (supernumerary derivative 22 syndrome): Clinical features of 63 individuals. Am J Med Genet A 149A: 1712-1721, 2009.

6. Benn P and Delach J: Human lymphocyte culture and chromosome analysis. CSH Protoc 2008: pdb. prot5035, 2008.

7. Xie L, Chen JL, Zhang WZ, Wang SZ, Zhao TL, Huang C, Wang J, Yang JF, Yang YF and Tan ZP: Rare de novo copy number variants in patients with congenital pulmonary atresia. PLoS One 9: e96471, 2014.

8. Tang M, Yang YF, Xie L, Chen JL, Zhang WZ, Wang J, Zhao TL, Yang JF and Tan ZP: Duplication of 10q22.3-q23.3 encompassing BMPR1A and NGR3 associated with congenital heart disease, microcephaly, and mild intellectual disability. Am J Med Genet A 167A: 3174-3179, 2015

9. Sun G, Tan Z, Fan L, Wang J, Yang Y and Zhang W: 1q21.1 microduplication in a patient with mental impairment and congenital heart defect. Mol Med Rep 12: 5655-5658, 2015.

10. Pinto D, Darvishi K, Shi X, Rajan D, Rigler D, Fitzgerald T, Lionel AC, Thiruvahindrapuram B, Macdonald JR, Mills R, et al: Comprehensive assessment of array-based platforms and calling algorithms for detection of copy number variants. Nat Biotechnol 29: 512-520, 2011.

11. Kurahashi H, Shaikh TH, Zackai EH, Celle L, Driscoll DA, Budarf ML and Emanuel BS: Tightly clustered 11q23 and 22q11 breakpoints permit PCR-based detection of the recurrent constitutional t(11;22). Am J Hum Genet 67: 763-768, 2000.

12. Biederman BM, Lin CC, Lowry RB and Somerville R: Tertiary trisomy (22q11q),47,+der(22),t(11;22). Hum Genet 53: 173-177, 1980.

13. Pihko H, Therman E and Uchida IA: Partial 11q trisomy syndrome. Hum Genet 58: 129-134, 1981.

14. Schinzel A, Schmid W, Auf der Maur P, Moser H, Degenhardt KH, Geisler M and Grubisic A: Incomplete trisomy 22. I. Familial 11/22 translocation with 3:1 meiotic disjunction. Delineation of a common clinical picture and report of nine new cases from six families. Hum Genet 56: 249-262, 1981.

15. Iselius L, Lindsten J, Aurias A, Fraccaro M, Bastard C, Bottelli AM, Bui TH, Caufin D, Dalprà L and Delendi N: The 11q;22q translocation: A collaborative study of 20 new cases and analysis of 110 families. Hum Genet 64: 343-355, 1983.

16. Lin AE, Bernar J, Chin AJ, Sparkes RS, Emanuel BS and Zackai EH: Congenital heart disease in supernumerary der(22), $\mathrm{t}(11 ; 22)$ syndrome. Clin Genet 29: 269-275, 1986.

17. Giraud F, Mattei JF, Mattei MG and Bernard R: Partial trisomy 11q and familial translocation 11-22 (author's transl). Humangenetik 28: 343-347, 1975 (In French).

18. Pangalos C, Couturier J, Bartsocas C and Theodorou S: Partial 11q trisomy due to missegregation of maternal $\mathrm{t}(11 ; 22)$ (q23; q11.1) translocation (author's transl). Nouv Presse Med 9: 3065-3067, 1980 (In French).

19. Mishra D, Kato T, Inagaki H, Kosho T, Wakui K, Kido Y, Sakazume S, Taniguchi-Ikeda M, Morisada N, Iijima K, et al: Breakpoint analysis of the recurrent constitutional $\mathrm{t}(8 ; 22)$ (q24.13;q11.21) translocation. Mol Cytogenet 7: 55, 2014.

20. Alberti A, Romano C, Falco M, Calì F, Schinocca P, Galesi O, Spalletta A, Di Benedetto D and Fichera M: $1.5 \mathrm{Mb}$ de novo 22q11.21 microduplication in a patient with cognitive deficits and dysmorphic facial features. Clin Genet 71: 177-182, 2007.

21. Courtens W, Schramme I and Laridon A: Microduplication 22q11.2: A benign polymorphism or a syndrome with a very large clinical variability and reduced penetrance?-Report of two families. Am J Med Genet A 146A: 758-763, 2008 .

22. Ensenauer RE, Adeyinka A, Flynn HC, Michels VV, Lindor NM, Dawson DB, Thorland EC, Lorentz CP, Goldstein JL, McDonald MT, et al: Microduplication 22q11.2, an emerging syndrome: Clinical, cytogenetic, and molecular analysis of thirteen patients. Am J Hum Genet 73: 1027-1040, 2003.

23. Lundin J, Söderhäll C, Lundén L, Hammarsjö A, White I, Schoumans J, Läckgren G, Kockum CC and Nordenskjöld A: 22q11.2 microduplication in two patients with bladder exstrophy and hearing impairment. Eur J Med Genet 53: 61-65, 2010. 
24. Zarate YA, Kogan JM, Schorry EK, Smolarek TA and Hopkin RJ A new case of de novo 11q duplication in a patient with normal development and intelligence and review of the literature. Am J Med Genet A 143A: 265-270, 2007.

25. Choi J, Lee H and Lee CG: Partial trisomy of 11q23.3-q25 inherited from a maternal low-level mosaic unbalanced translocation. Am J Med Genet A 167A: 1859-1864, 2015.

26. Göhring I, Blümlein HM, Hoyer J, Ekici AB, Trautmann U and Rauch $\mathrm{A}$ : $6.7 \mathrm{Mb}$ interstitial duplication in chromosome band 11q24.2q25 associated with infertility, minor dysmorphic features and normal psychomotor development. Eur J Med Genet 51: 666-671, 2008.
27. Burnside RD, Lose EJ, Domínguez MG, Sánchez-Corona J, Rivera H, Carroll AJ and Mikhail FM: Molecular cytogenetic characterization of two cases with constitutional distal 11q duplication/triplication. Am J Med Genet A 149A: 1516-1522, 2009.

28. Kayhan G, Cavdarli B, Yirmibes Karaoguz M, Percin EF, Oztürk Kaymak A, Biri A and Ergun MA: Molecular karyotyping of an isolated partial trisomy 11q patient with additional findings. Gene 524: 355-360, 2013

29. Ben-Abdallah-Bouhjar I, Mougou-Zerelli S, Hannachi H, Ben-Khelifa H, Soyah N, Labalme A, Sanlaville D, Elghezal H and Saad A: Phenotype and micro-array characterization of duplication 11q22.1-q25 and review of the literature. Gene 519: 135-141, 2013. 